\title{
Exploiting the HASH Planetary Nebula Research Platform
}

\author{
Quentin A. Parker ${ }^{1,2}$, Ivan Bojičićc ${ }^{1,2}$, David J. Frew ${ }^{1,2}$ \\ ${ }^{1}$ The University of Hong Kong, Department of Physics, Hong Kong SAR, China \\ ${ }^{2}$ The University of Hong Kong, Laboratory for Space Research, Hong Kong SAR, China \\ email: quentinp@hku.hk
}

\begin{abstract}
The HASH (Hong Kong/ AAO/ Strasbourg/ H $\alpha$ ) planetary nebula research platform is a unique data repository with a graphical interface and SQL capability that offers the community powerful, new ways to undertake Galactic PN studies. HASH currently contains multi-wavelength images, spectra, positions, sizes, morphologies and other data whenever available for 2401 true, 447 likely, and 692 possible Galactic PNe, for a total of 3540 objects. An additional 620 Galactic post-AGB stars, pre-PNe, and PPN candidates are included. All objects were classified and evaluated following the precepts and procedures established and developed by our group over the last 15 years. The complete database contains over 6,700 Galactic objects including the many mimics and related phenomena previously mistaken or confused with PNe. Curation and updating currently occurs on a weekly basis to keep the repository as up to date as possible until the official release of HASH v1 planned in the near future.
\end{abstract}

Keywords. Planetary nebulae, multi-wavelength imagery, spectroscopy, databases, late-stage stellar evolution

\section{Introduction}

Planetary Nebulae (PNe) are a useful tracer and touchstone population for studies of late-stage stellar evolution. Their strong emission lines permit the size, expansion velocity and age of the PN to be determined. We can use them to determine the chemical abundance pattern of the ejected gas while their varied forms provide clues to their formation, evolution, mass-loss processes, and possible shaping mechanisms. Such properties make PNe ideal age, abundance, and kinematic probes. Much of this power comes from statistical studies of PNe. Even some recent studies (e.g. Stanghellini \& Haywood 2011) have relied on unrepresentative flux-limited samples taken from the early catalogues of Acker et al. $(1992,1996)$ and Kohoutek (2001). We now have a better understanding of the true underlying PN population within the Galaxy, with the discovery of many hundreds of PNe that are fainter, more obscured, and more evolved, as illustrated by the MASH survey (Parker et al. 2006; Miszalski et al. 2008). Earlier compilations were affected by significant numbers of contaminants that have degraded inferences and results from such studies (e.g. Cohen et al. 2011).

This situation has changed and a range of techniques have now been developed to effectively remove PN mimics (e.g. Frew \& Parker 2010). There is a need to consolidate and re-evaluate the PN population from earlier catalogues in light of these tools, combined with the significant number of new discoveries (Frew 2016, these proceedings). We also undertook new measurements of known PNe from the latest available high-resolution, multi-wavelength imaging surveys. We now provide the community with a more robust master catalogue of Galactic PNe that offers the best possible data available to undertake statistical investigations on the Galactic PN population. This repository is known as the 


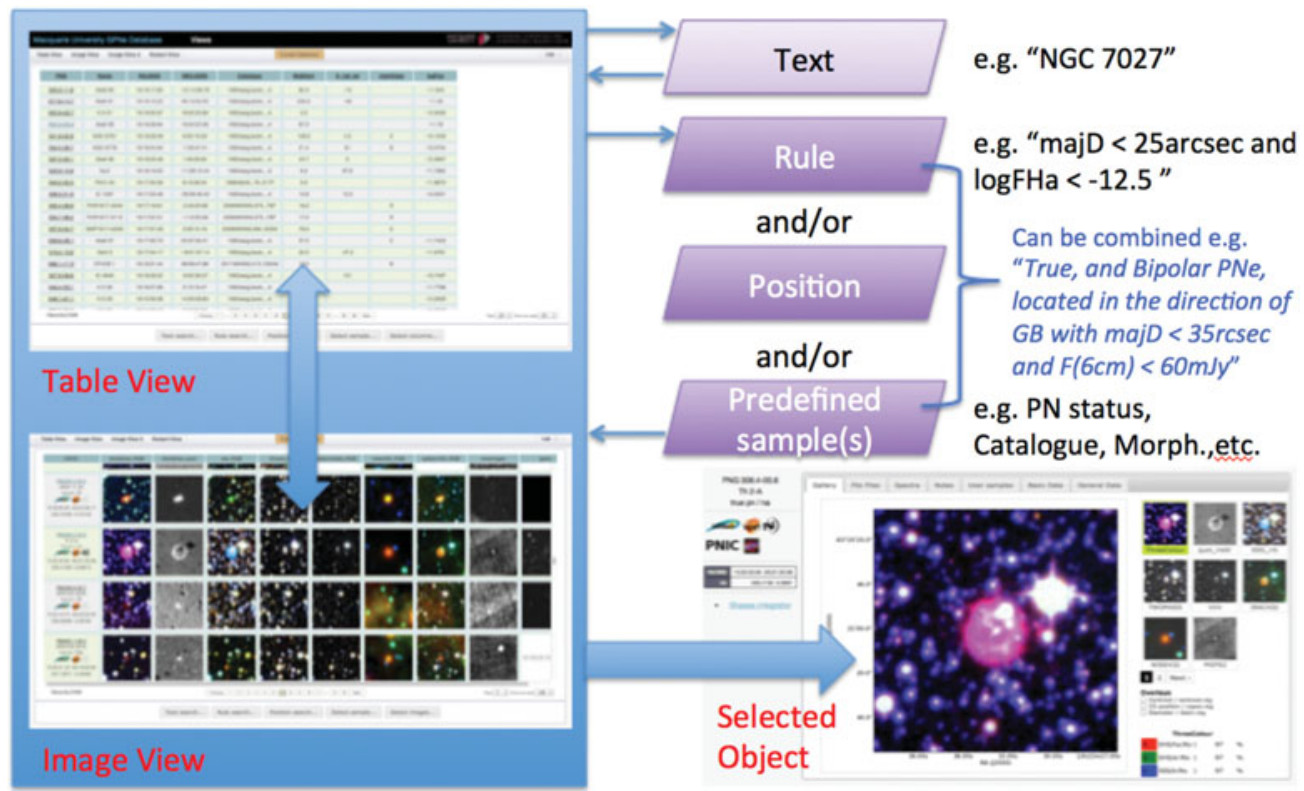

Figure 1. Example of the process by which the HASH catalogue can be visualised, first as a table of selected objects, then as a set of selected images, and then as an individual page for a single $\mathrm{PN}$, presenting key data. It also shows how predefined samples selected according to user-applied rules can be extracted for further study.

HASH (Hong Kong/ AAO/ Strasbourg/ H $\alpha$ ) PN research platform (Parker, Bojičić \& Frew 2016) and is recommended for use for all PNe studies.

\section{Background}

Over the last 10 years there has been a significant increase in the numbers of Galactic PNe known thanks in particular to the narrowband $\mathrm{H} \alpha$ Galactic plane surveys of Parker et al. (2005), and Drew et al. (2005, 2014). These offer exceptional discovery potential as reported in the MASH catalogues and IPHAS catalogues (e.g. Sabin et al. 2014). The amateur community, including the Deep Sky Hunters consortium, has also been actively trawling digitised broadband and narrowband surveys to reveal over a hundred new PN candidates (Jacoby et al. 2010; Kronberger et al. 2016), many of which have now been spectroscopically confirmed. Significant numbers of candidates have also been revealed by their multi-band characteristics in the mid-IR (Cohen et al. 2011) or radio (Hoare et al. 2012) domains. As most are heavily obscured, spectroscopic confirmation is difficult. In some cases PN candidates identified purely on their mid-IR and radio properties do have faint optical counterparts and have now been spectroscopically confirmed (e.g. Parker et al. 2012; Fragkou et al. 2016, these proceedings).

The HASH research platform brings all these different samples together into the same repository and attempts to apply the same rigorous set of parameter estimations for all. Full details of the scope, organisation, and utility of HASH will be presented in a refereed paper currently in train (Bojičić et al., in preparation). In this brief conference paper only a little of the power and flexibility of this facility is described, as shown in Figure 1. 
HASH PN Database4.2 / PN Info / PNG 165.5-06.5

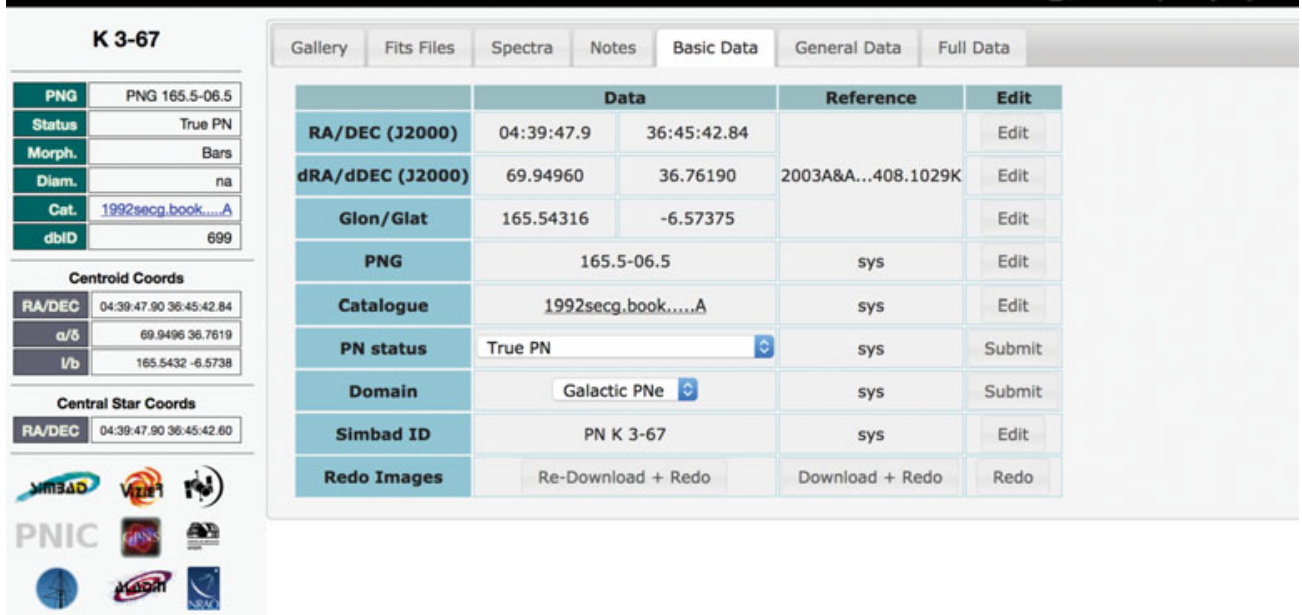

Figure 2. HASH page showing content under the basic data tab for the true PN K 3-67. Note the clickable icons at bottom left which provide a direct link to that object in various external databases, including Simbad, Vizier, MAST, and the San-Pedro Martir database (Lopez et al. 2012), etc. A highlighted icon indicates data are available from these external resources.

\section{What can HASH do?}

HASH (hashpn.space) is an integrated online system that is extremely flexible. It allows any registered user access not only to the best catalogue of Galactic PNe ever compiled, but also offers a powerful research platform to visualise and extract data for analysis from the database according to any valid SQL query. This can currently be done on more than 30 columns of data including positions (RA/Dec and $l, b$ ), usual names, sizes, morphologies, original catalogue origin, and many more. All entries have been checked, verified and in most cases re-measured from the latest imaging surveys in an attempt to provide the most robustly determined data possible. Many of these data columns refer to measured properties such as optical narrowband, mid-IR, or radio photometry whose values are being incorporated into HASH.

Additionally, wherever possible, optical (and some near-IR) spectra are included. Spectra are a pre-requisite for any PN to be classed as T (true). Thousands of these spectra come from our own follow-up observations on a series of mainly 2-metre class telescopes made over the last 15 years. A further 1050 low-resolution spectra of varying quality are also made available in HASH for the first time from the Stenholm-Acker survey (Stenholm \& Acker 1987). Published emission-line fluxes from ELCAT, converted into line plots are also available for 1020 PNe (see Kaler, Shaw \& Browning 1997). HASH includes a spectral visualisation tool, where the user can highlight various emission lines, choose and over-plot multiple spectra when available, and expand these spectra on screen; the user can also download all the 1-D fits spectra for more careful measurement and analysis. Furthermore, the user can download all the individual WCS fits files comprising the multi-wavelength color images, all put on a common astrometric grid as far as possible. An additional feature of HASH is the incorporation of an interactive flux integrator when suitable image data are available from the SHS, SHASSA, or VTSS H $\alpha$ surveys (we plan to add IPHAS and VPHAS+ images in the near future). This tool offers users the capacity to undertake their own flux estimations following Frew, Bojičić \& Parker (2013) and Frew et al. (2014), who estimated H $\alpha$ fluxes for over 1300 PNe. 


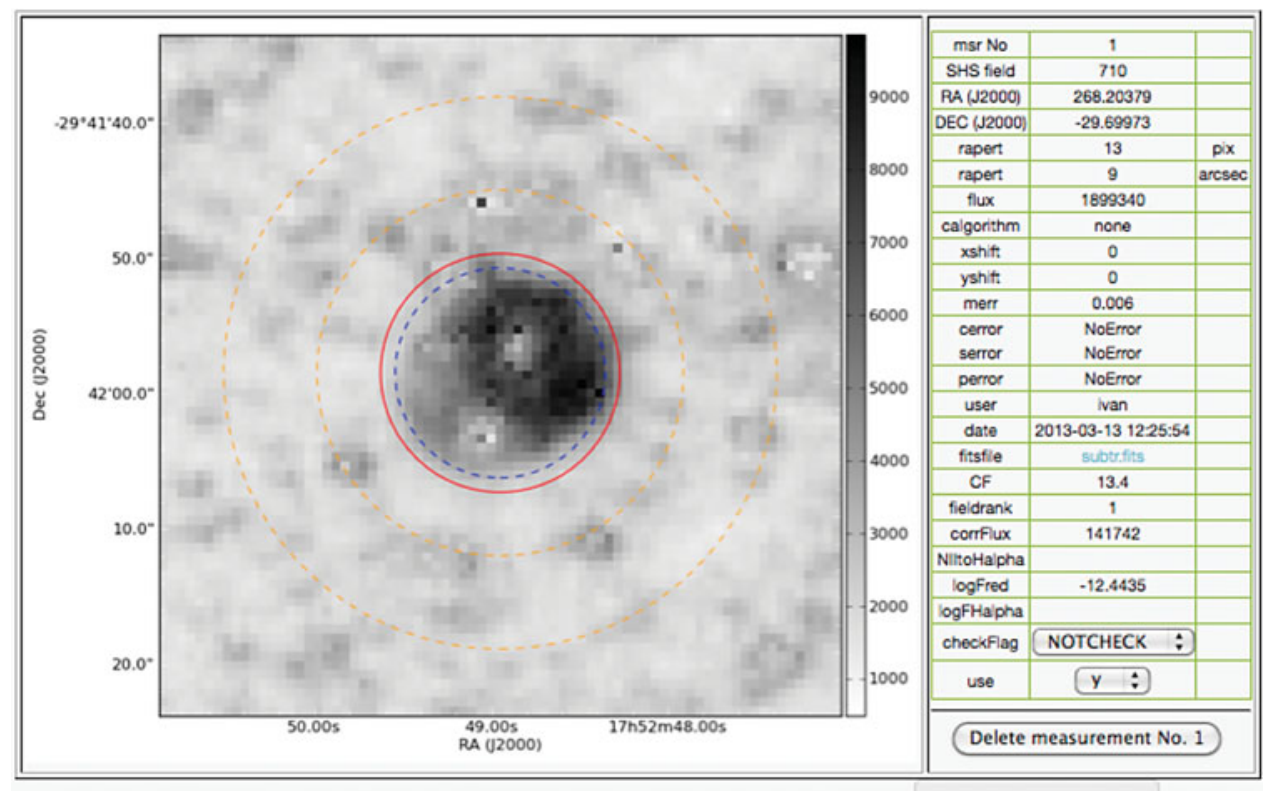

Add Measurement

Figure 3. HASH page showing the interactive flux integrator that is available.

\section{References}

Acker, A., et al. 1992, Strasbourg-ESO Catalogue of Galactic Planetary Nebulae. Garching Acker, A., Marcout, J., \& Ochsenbein, F. 1996, First Supplement to the SECGPN. Strasbourg Cohen, M., et al. 2011, MNRAS, 413, 514

Drew, J. E., et al. 2005, MNRAS, 362, 753

Drew, J. E., et al. 2014, MNRAS, 440, 2036

Fragkou, V., Bojičić, I., Frew, D., \& Parker, Q. 2016, these proceedings (arXiv.1611.08590)

Frew, D. J. 2016, these proceedings (arXiv preprints)

Frew, D. J. \& Parker, Q. A., 2010, PASA, 27, 129

Frew, D. J., Bojičić, I. S., \& Parker, Q. A. 2013, MNRAS, 431, 2

Frew, D. J., et al. 2014, MNRAS, 440, 1080

Hoare M. G., et al. 2012, PASP, 124, 939

Jacoby, G. H., et al. 2010, PASA, 27, 156

Kaler, J. B., Shaw, R., \& Browning, L. 1997, PASP, 109, 289

Kohoutek, L., 2001 A $\& A, 378,843$

Kronberger, M., et al. 2016, JPhCS, 728, 072012

López, J. A., et al. 2012, Rev. Mex. Astron. Astrofís., 48, 3

Miszalski, B., et al. 2008, MNRAS, 384, 525

Parker, Q. A., et al. 2005, MNRAS, 362, 689

Parker, Q. A., et al. 2006, MNRAS, 373, 79

Parker, Q. A., et al. 2012, MNRAS, 427, 3016

Parker, Q. A. \& Bojičić, I. S., Frew D. J. 2016, JPhCS, 728, 032008

Sabin, L., et al. 2014, MNRAS, 443, 3388

Stanghellini, L. \& Haywood, M. 2010, ApJ, 714, 1096

Stenholm B. \& Acker, A. 1987, A\&AS, 68, 51

\section{Discussion}

StAnghellini: Please use the SIMBAD PNG nomenclature in your catalogs. What is the actual number of spectroscopy-confirmed Galactic planetary nebulae? 